\title{
Primary Non Hodgkins Lymphoma of Bilateral Breasts: A Rare Entity
}

\author{
Gireesha Rawal*, Sufian Zaheer, AK Yadav and AK Mandal \\ Dept. of Pathology, Vardhman Mahavir Medical College, Delhi, India
}

\section{ABSTRACT}

Extranodal Non-Hodgkin's Lymphoma (NHL) of the breast is a rare entity, representing $0.04-1.1 \%$ of malignant tumours of the breast. Most breast lymphomas are the non-Hodgkin's B cell type, with DLBCL being the most common. We present the case of a 32 years old female who presented with bilateral hard breast lumps, which were clinicoradiologically thought to be carcinomas. But on histopathology and immunohistochemistry, it was finally diagnosed as a lymphoma. Primary and secondary lymphomas of the breast, though rare, should be considered in the differential diagnosis of breast malignancies.

Keywords: Breast, Lymphoma, NHL

\section{Introduction}

Extranodal Non-Hodgkin's Lymphoma (NHL) of the breast is a rare entity. It constitutes $0.04-1.1 \%$ of malignant tumours of the breast, $1.7-2.2 \%$ of extranodal lymphomas and $0.7 \%$ of all NHL. ${ }^{[1]}$ However, primary NHL (PNHL) is the most frequent hematopoetic tumour of breast ${ }^{[1]}$. Mucosa associated lymphoid tissue (MALT) lymphoma is another common type of breast lymphomas. ${ }^{[2]}$

Non-Hodgkin's typebreast lymphoma represents approximately $70-90 \%$ of breast lymphomas. In patients diagnosed with NHL, primary involvement of the breast is seen in $0.4-0.7 \%$ of the cases. ${ }^{[3]}$ Almost all primary breast lymphomas have a B-cell phenotype, while primary breast lymphomas with a T-cell phenotype are extremely rare. [4] $46-71 \%$ of primary breast lymphomasare diffuse large B-cell lymphomas (DLBCL). ${ }^{[5,6]}$ Primary breast lymphoma exhibits a poor prognosis.and the therapeutic management is controversial and is not fully established.

\section{Case report}

A 32-year-old woman presented with a hard mass in the bilateral breasts for 3 months. Mammography showed a diffuse increase in the density of the breasts. Other investigations were unremarkable. Clinicoradiologically, it was suspected to be carcinoma breast. Biopsy was done from bilateral lesions and histopathological picture was similar showing sheets of dyscohesive tumour cells entirely replacing the normal breast tissue. The tumour cells were large, round with moderate to scanty cytoplasm. Tumour cells were showing severe degree of pleomorphism. High degree of mitosis could be identified.

Differential diagnosis of poorly differentiated carcinoma and lymphoma were considered. Immunohistochemistry panel of ER, PR, Her2neu, Ki-67, LCA, CK and EMA was put up. LCA, EMA and Ki-67 came out to be positive, and rest of the markers were negative. This directed us towards lymphoma.A final diagnosis of NHL was given.

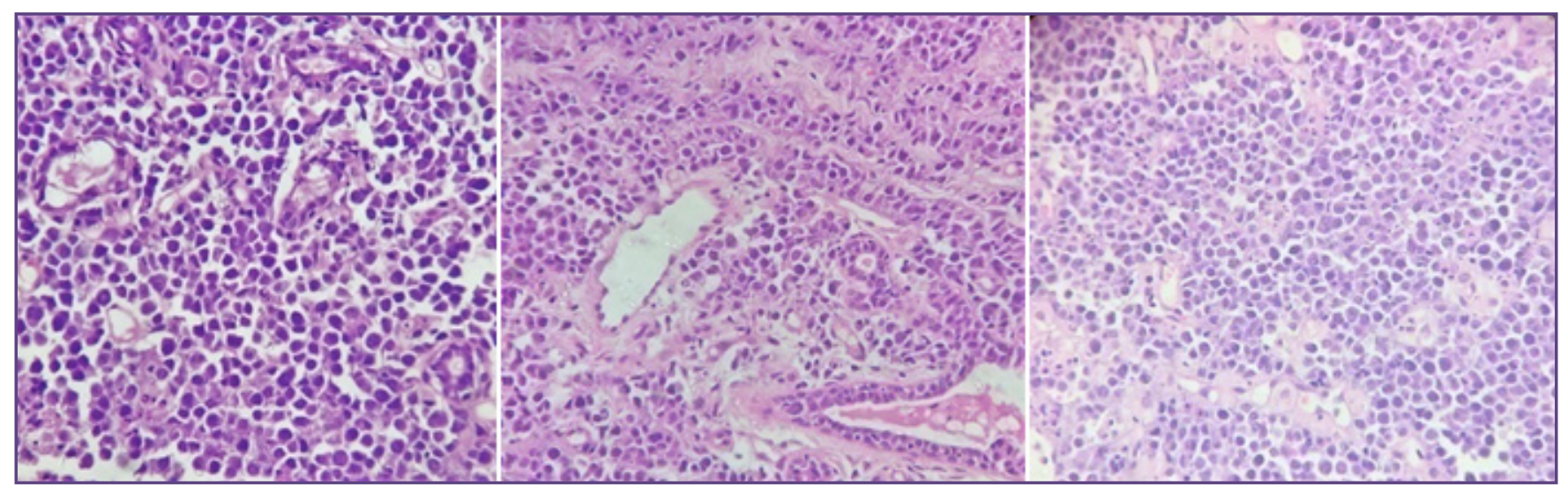

Fig. 1,2,3: H\&E showing features of NHL (40X). 


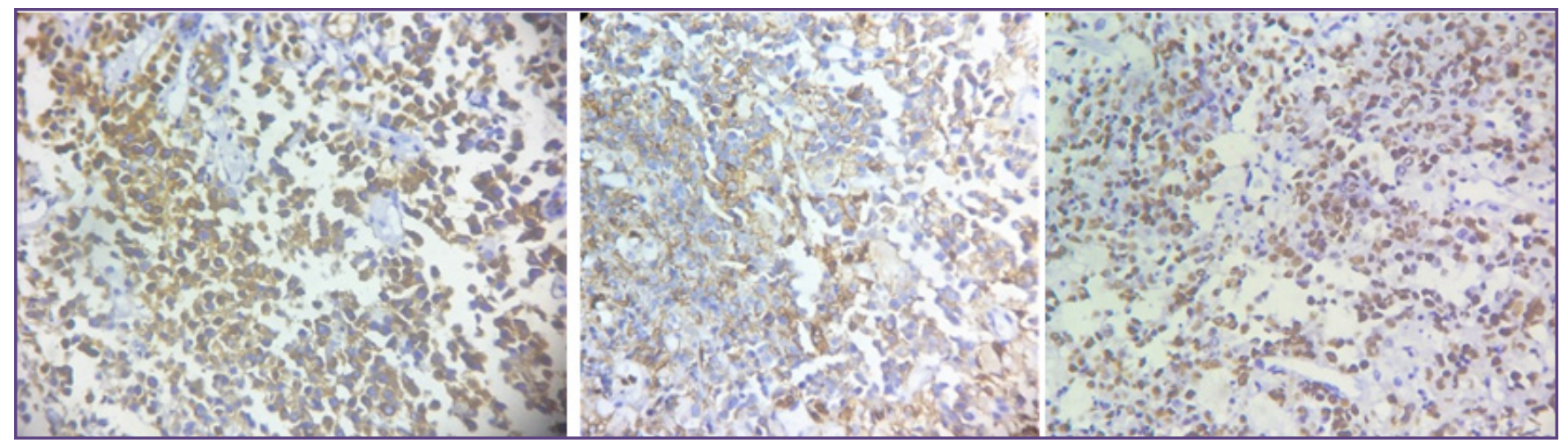

Fig. 4 A: IHC showing positivity of LCA (40X), B: positivity of EMA (40X); C: high positivity index of Ki-67 (40X)

\section{Discussion}

NHL involving the breast either as a primary site or as a site of recurrence from lymphoma previously diagnosed elsewhere is rare. Several series have reported varying incidences of primary and secondary cases. Primary NHL of the breast is a rare entity, constituting only $0.04 \%-0.50 \%$ of malignant breast neoplasms, $1.7 \%$ of all extranodal NHL and $0.7 \%$ of all NHL. ${ }^{[1]}$

Clinically, primary breast lymphoma presents with features similar to that of breast carcinoma. It usually presents with right sided painless lump, sometimes multinodular, and which can be bilateral in $10 \%$ of cases. ${ }^{[7]}$ Our patient had bilateral breast involvement. It affects two distinct age groups, one which affects a young woman is frequently bilateral, often associated with pregnancy, and is Burkitttype lymphoma. The second group affects older women and is usually unilateral. ${ }^{[6,8]}$

Since there is considerable overlap in clinical and radiological features of breast lymphoma and carcinoma, pathology remains gold standard to differentiate these two malignancies. Although sensitivity of FNAC in diagnosis of lymphoproliferative disorder is $90 \%$, several diagnostic pitfalls exist. Confirmatory core needle biopsy is recommended for a suspected primary lesion. ${ }^{[4]}$

Histologically, primary breast lymphomas are predominately of B-cell origin and most commonly large cell type. ${ }^{[9]}$ The rate of secondary lymphoma metastatic to breast only slightly exceeds primary breast involvement. ${ }^{[9,10]}$

The following strict criteria must be met for a neoplasm to be characterized as PNHBL: (1) an adequate pathologic specimen, (2) close association of mammary tissue and lymphomatous infiltrate, (3) no evidence of disseminated lymphoma at the time of diagnosis, and (4) involvement of ipsilateral axillary nodes only if it occurs concomitantly with the primary lesion. ${ }^{[4]}$ In our case, all these criteria were met for the diagnosis of PNHBL.

The histological differential diagnosis of breast lymphomas include lobular carcinoma, medullary carcinoma, amelanotic melanoma and poorly differentiated duct carcinoma. IHC and/or flow cytometry is helpful in differentiating these. In addition to physical examination, radiology of the chest, skull and pelvis is a reliable method for detecting visceral and nodal dissemination, and should always be performed.Contralateral breast involvement is best ruled out by MRI scan. It is also useful in follow up of patients to monitor response to chemotherapy and radiotherapy and to diagnose disease recurrence. The risk of CNS relapse in patients with primary breast lymphoma is greater than that of aggressive nodal NHL, and approximately estimated as $5 \% .^{[1]}$

The treatment of PNHBL is similar to that of other lymphomas and depends on the histological typeand histologic grade. Patients with low grade disease can be managed with local therapy alone. Patients with intermediate or high grade disease have better outcome if chemotherapy is included. Recent studies have shown that aggressive B-cell lymphomas should always be treated with chemotherapy alone or in combination with radiotherapy. The most effective combination reported in literature is radiotherapy and 3 to 10 cycles of $\mathrm{CHOP}$ regime.

Overall 5 year survival rate is $43 \% \cdot{ }^{[12]}$ Survival rate of primary breast lymphoma is better as compared to systemic lymphoma with secondary breast involvement. [4] Anticancer drugs are the main treatment rather than surgery, so it is very important to accurately diagnose primary lymphoma of breast.

\section{Conclusion:}

In summary, we report a case of primary lymphoma of bilateral breasts, which was clinically andradiologically 
suspected to be a carcinoma. Histologically, the tumour demonstrated the characteristic histopathological and IHC features of a lymphoma. Primary and secondary lymphomas of the breast, though rare, should be considered in the differential diagnosis of breast malignancies.

\section{References:}

1. Sinha A, Ramrakhiani D. Primary non-Hodgkin's lymphoma of the breast: a case report. ActaCytol. 2005;49:661-665.

2. Chakraborty J, Paul PC, Sarkar R, Rao RN. Primary nonHodgkin's lymphoma of breast: a case report. Indian J PatholMicrobiol. 2007;50:315-317.

3. Shukla S, Awasthi NP, Singh P, Husain N. CD20 negative primary diffuse large B cell lymphoma of breast: Role of Pax-5. J Can Res Ther. 2015;11:658.

4. Nigam A, Singh AK, Singh SK, Singh N, Singh N, Sharma A. Primary Mammary (Non-Hodgkin) Lymphoma of Breast: A Case Report. J Cancer SciTher. 2011;3:173-175.

5. Cohen Y, Goldenberg N, Kasis S, Shpilberg D, Oren M. Primary breast lymphoma. Harefuah. 1993;125:24-26.

6. Jeon HJ, Akagi T, Hoshida Y, Hayashi K, Yoshino T, Tanaka $\mathrm{T}$, et al. Primary non-Hodgkin malignant lymphoma of the breast. An immunohistochemical study of seven patients and literature review of 152 patients with breast lymphoma in Japan. Cancer. 1992; 70:2451-9.

7. Lamovec J, Wotherspoon A, Jacquemier J. Malignant lymphoma and metastatic tumours. In: Tavassoli FA, Devilee P, editors. World health organisation classification of tumors: pathology and genetics : tumours of the breast and female genital organs. Lyon: IARC; 2003.107-109.

8. Bobrow LG, Richard MA, Happerfield LC, Diss TC, Isaacson PG, Lammie GA, et al. Breast lymphomas: a clinicopathologic review. Hum Pathol. 1993;24:274-278.

9. Darnell A, Gallardo X, Sentis M, Castañer E, Fernandez E, Villajos M. Primary lymphoma of the breast: MR imaging feature. A case report.MagnReson Imaging. 1999; 17:479-482.

10. Paulus DD. Lymphoma of the breast.RadiolClin North Am. 1990;28:833-840.

11. Hill QA, Owen RG. CNS prophylaxis in lymphoma: who to target and what therapy to use. Blood Rev. 2006;20:319-332.

12. Roberto G, Piccollo C, Rilke F. Primary Non Hodgkin's lymphoma of the female breast. Cancer. 1992;69:725735. See comment in PubMed Commons below

*Corresponding author:

Dr. Gireesha Rawal, Dept. of Pathology, Vardhman Mahavir Medical College, Delhi, India

Email: gireesharawal@yahoo.in

Date of Submission : 12.02.2017

Date of Acceptance : 15.06.2017

Financial or other Competing Interests: None.

Date of Publication : 19.10.2017 